\title{
11) Nachricht für diejenigen, welche Droguen und medicinische Präparate nach den Vereinigten Staaten senden wollen.
}

1. Art. Der Senat und die Kammer der Reprăsentanten der Vereinigten Staaten, in pleno versammelt, decretiren, dass vom Dato der Verkündigung der gegenwärligen Artikel an, alle in den Vereinigten Staaten eingebrachte Droguen, sie mögen in medicinischen Prâparaten, ătherischen 0elen, oder in als Arzneien gebräuchlichen chemischen Präparaten bestehen, ehe sie aus der louane entlassen werden, einer Prüfung, wodurch ihre Güte, Reinheit und ihre Anwendung zum ärztlichen Gebrauch, wie auch ihr Werth und ihre Identität in Uebereinstimmung mit dem Bülletin der Expedition bewabrheitet wird, zu unterwerfen sind.

2. Art. Alle medicinischen, chemischen und andern Prïparate, welche gewöhnlich mit dem Namen des Manufacturisten verseben angebracht sind, müssen diesen Namen gehörig beglaubigt enthalten, wio auch den Ort der Fabrication, auf einer sehr leserlichen Etiquelte, ferner Steinpel und sonstige Atteste mit sich führen, Alle Präparate u. s. w., die dieser Formalität entbehren, werden zur Conliscation ausgeschlossen.

3. Art. Wenn besagte Droguen u.s. w. bei der Prüfung als verfälscht, oder in der Art verschlechtert erkannt sind, dass sic hinsichtlich ihrer Stärke und Reinheit denen in den Pharmakopöen der Vereinigten Staaten, Edinburgs, Londons, Frankreichs und Deutschlands aufgenommenen nicht enlsprechen, folglich gefährlich oder zum medicinischen Gebrauch untauglich erscheinen, so muss darüber ein Bericht erfolgen. Gedachte Gegenstănde dürfen dann von den Dounnen nicht abgefordert werden, wenn nicht eine vom Eigenthümer etc. verlangte neue Prüfung die erste als mangelhaft erweist und zeigt, dass die in Rede stehenden Mledicamente als solche ohne Gefahr angewandt werden können.

4. Art. İst der Eigenthūmer oder der designirte Empfänger mit der ersten Prüfung nicht zufrieden, so soll er das Recht haben, eine erneuerte Untersuchung anf seine losten zu verlangen, und nachdem er eine hinreichende Summe als Caution deponirt hat, ihn ein erfahrener Chemiker aus den Collegien der Pharmacie und Medicin angewiesen werden, welcher eine genaue chemische Prüfung der erwähnlen Gegenstände bewerkstelligen und dieselbe an Eidesstatt bekräftigen muss. In dem Fall, diss dieser zweite entscheidende Bericht Fehler des ersten aufwiese, wie auch, dass die untersuchten Gegenstände den oben angedeuteten entsprechen, so wird die Sendung angenommen, und zwar unter Vorbehalt der gewöhnlichen Douanenkosten. Bestätigt aber der zweite Bericht den ersten, so bleiben die in Rede stehenden Geginstände in den Hånden des Collecteurs, und der Eigenthümer etc. darf, nacbdem er sich der Magazin- und anderer Kosten entledigt hat, und sich verbindlich macht, die Waaren innerhalb sechs Monaten - von der Untersuchungszeit an - wieder üher die Grenze der Vereiniglen Staaten za schaffen, sie wieder zurückschicken.

Ist nach Ablauf der bemerkten Zeit die Waare noch nicht über die Grenze, so ist der Magazinaufseber verpflichtet, sie zu zernichten, wobei er den Eigenthümer oder designirten Empfanger verbindlich macht, die Kosten der wirklichen Ausführung zu stehen. 
5. Art. Zur Erfüllung obiger Zwecke ist der Secretair des Schatzes ernächligt, die nöthigen Summen zur Besoldung einer gewissen Anzahl erfahrener, zur Pröfung angewiesener Chemiker zurückwhalten; namentlich für die Häfen von Newyork, Bosıon, Philadelphia, Charlestown, New-Orleans elc. Er wird auch zugleich den Einnehtuern der Douanen die zur Entfernung verfalschter Droguen und medicinischer I'rjparate von ilın nöthig erachteten Instructionen geben.

Die übrigen Artikel dieses Decrets bezichen sich auf administrative Massregeln, die zur Befolgung obiger Statute za nehmen sind.

Das Collegium der Pharnacie hat bei seiner Sitzung am 8 . Juli 1548 Ansichten veröfentlicht, wodurch die Ausführung des obigen Decrets erleichtert werden kann, und Copien davon an alle medicinische und pharmaceulische Collegien, wie auch an die Redaction der Pharnaceutical Times and Journal of chemestry, ferner das Pharmacculical Journal and Transaction Londons, endlich das Journal de Pharmacie in Paris gesandt.

Wer sicht es nichl, wie wünschenswerth es wïre, dass deutsche Einigkeit 24 ithnlichen Masssregeln in den Stehüfeı etc. Gelegenheit gäbe.

d. il.

\section{2) Handelsbericht von}

Schubart \& Bade.

Ilamburg, den 6. März 1849.

Bei dem Wicderbeginn des Frühlings, wo der Handel durch die hergestellicn Communicationen nach allen Richtungen ein reges Leben annimmt, freut es uns in der That, diese'm Zeitpuncte nit schöncren Hoffnungen näher zu lommen, als man solches nach den Weltereignissen des vorigen Jahres, die eine grosse Rolle in der Geschichte cinnehmen, zu erwarten bereclitigt war. Die Errungenschaften, dio seit dem vorigen Frühjahre auftauchlen und sich der Gemüther beinichtigten, waren zu aufregender Arl, als dass Handel und Gewerbe in gewohntem, ruhigen Fortgange bleiben konnten; wie sehr diese darnieder lagen und Misstrauen sich von allen Seiten kund gab, darüber ist wolıl nur eine Stimme. In vollen Vertrauen, dass die bedeulungsvolle $Z$ eil, in der wir uns befinden, auf ruhige, vernünftige Weise benutzt wird, liegen wir die feste IIofhung, dass solches wohlihälig und befördernd auf llandel nnd (iewerbe überhaupt influiren muss.

In unsermI Droguen-Narkt haben seit unsern letzten ausführlichen Mittheilungen yom 1 October a. p, sehr viele Artikel eine steigende Tendenz angenommen und bleiben in solcher J'osition, die auch ferner ils günstig zu liezeichnen ist; namentlich sind dartunter die ostindischen Jroguen zu zililen, welche bekanntlich den Importeuren seit Jahr und Tag, durch forlwäbrende Reaction, nambafte Verluste zuführten. Bei der durehweg reellern Basis, welche der llandel im Allgemeinen angenommen hat, wird auch in unserer Branche mancher Arlikel einen hüheren Werth annehmen, der jetat noch ohme Berücksichtigung liegt. - Durch nachstehenden Warenbericht erlauben wir uns, Ihien so genau als möglich einen getreucn Ueherblick über unsern DrogucnHarkt zu verschaffen, führen Ihnen die beachtenswerthen Gegenstande einzeln vor und lassen zum Schluss einige lotails über Artikel folgen, welche zu unserer Branche gehören und worübcr uns ein angesehenes llaus in London nähere vescription gab, wünschend, dass sowohl 